\title{
SOME FIXED POINT THEOREMS IN MENGER PROBABILISTIC PARTIAL METRIC SPACES WITH APPLICATION TO VOLTERRA TYPE INTEGRAL EQUATION
}

\author{
AMIR GHANENIA, MAHNAZ KHANEHGIR, REZA ALLAHYARI* AND MOHAMMAD \\ MEHRABINEZHAD \\ Department of Mathematics, Mashhad Branch, Islamic Azad University, Mashhad, Iran \\ *Corresponding author: rezaallahyari@mshdiau.ac.ir

\begin{abstract}
In this paper, we introduce the notion of Menger probabilistic partial metric space and prove some fixed point theorems in the framework of such spaces. Some examples and an application to Volterra type integral equation are given to support the obtained results. Finally, we apply successive approximations method to find a solution for a Volterra type integral equation with high accuracy.
\end{abstract}

\section{INTRODUCTION}

The concept of a Menger probabilistic metric space (briefly, Menger PM-space) was initiated by Menger [15]. The idea of Menger was to use a distribution function instead of a nonnegative number for the value of a metric.

The study of this space was expanded rapidly with the pioneering works of Schweizer and Sklar [20], Stevens [25] and some of their coworkers. In 1972, Sehgal and Bharucha-Reid [23] obtained a generalization of the Banach contraction principle on a complete Menger space. Since then, a number of mathematicians have made a substantial contribution to the theoretical development of Menger PM-spaces (see $[4,6-10,12,17,26]$ ).

Received 2019-04-19; accepted 2019-08-14; published 2019-09-02.

2010 Mathematics Subject Classification. 47H10, 54H25.

Key words and phrases. Fixed point; Menger probabilistic metric space; Partial metric space; Volterra type integral equation; Successive approximations method.

(C)2019 Authors retain the copyrights of their papers, and all open access articles are distributed under the terms of the Creative Commons Attribution License. 
On the other hand, Matthews [14] introduced the notion of partial metric space as a part of the study of denotational semantics of dataflow networks. In recent years, some scholars have investigated the topological properties of partial metric spaces and have established some fixed point results in these spaces (see $[1,3,5$, $16,19,22])$.

In this work, using the concepts of Menger probabilistic metric space and partial metric space, we establish a new concept of Menger probabilistic partial metric space. We present some fixed point theorems in these spaces. Some examples and an application to Volterra type integral equation are given to illustrate the usability of our results. Finally, we apply successive approximations method to find an approximate solution for a Volterra type integral equation with high accuracy.

First, we recall some basic definitions and facts which will be used further on. We denote by $\mathbb{R}$ the set of real numbers and $\mathbb{R}^{+}$the set of nonnegative real numbers.

Definition 1.1. [14] A partial metric on a nonempty set $X$ is a mapping $p: X \times X \rightarrow \mathbb{R}^{+}$such that for all $x, y, z \in X:$

(p1) $x=y$ if and only if $p(x, x)=p(x, y)=p(y, y)$,

(p2) $p(x, x) \leq p(x, y)$,

(p3) $p(x, y)=p(y, x)$,

(p4) $p(x, y) \leq p(x, z)+p(z, y)-p(z, z)$.

A pair $(X, p)$ is called a partial metric space, if $X$ is a nonempty set and $p$ is a partial metric on $X$.

It is clear that if $p(x, y)=0$, then from (p1) and (p2), $x=y$. But if $x=y, p(x, y)$ may not be 0 . A basic example of a partial metric space is the pair $\left(\mathbb{R}^{+}, p\right)$, where $p(x, y)=\max \{x, y\}$ for all $x, y \in \mathbb{R}^{+}$(see [16]).

Each partial metric $p$ on a nonempty set $X$ generates a $T_{0}$ topology $\tau_{p}$ on $X$ whose base is the family of open $p$-balls $\left\{B_{p}(x, \varepsilon): x \in X, \varepsilon>0\right\}$, where $B_{p}(x, \varepsilon)=\{y \in X: p(x, y)<\varepsilon+p(x, x)\}$ for all $x \in X$ and $\varepsilon>0$.

Definition 1.2. [14] Let $(X, p)$ be a partial metric space, $\left\{x_{n}\right\}$ be any sequence in $X$ and $x \in X$. Then:

(i) The sequence $\left\{x_{n}\right\}$ is said to be convergent to $x$ with respect to $\tau_{p}$ if $\lim _{n \rightarrow \infty} p\left(x_{n}, x\right)=p(x, x)$.

(ii) The sequence $\left\{x_{n}\right\}$ is said to be a Cauchy sequence in $(X, p)$, if $\lim _{n, m \rightarrow \infty} p\left(x_{n}, x_{m}\right)$ exists and is finite.

(iii) $(X, p)$ is said to be a complete partial metric space if for every Cauchy sequence $\left\{x_{n}\right\}$ in $X, x \in X$ exists such that

$$
\lim _{n, m \rightarrow \infty} p\left(x_{n}, x_{m}\right)=\lim _{n \rightarrow \infty} p\left(x_{n}, x\right)=p(x, x) .
$$

Notice that in a partial metric space the limit of a convergent sequence may not be unique. 
In the sequel, we recall the definition of $\mathrm{n}$-th order t-norm. Shi et al. [24] gave the following definition of n-th order t-norm.

Definition 1.3. A mapping $T: \prod_{i=1}^{n}[0,1] \rightarrow[0,1]$ is called an $n$-th order $t$-norm if the following conditions are satisfied:

(i) $T(0,0, \ldots, 0)=0, T(a, 1,1, \ldots, 1)=a$ for all $a \in[0,1]$,

(ii) $T\left(a_{1}, a_{2}, a_{3}, \ldots, a_{n}\right)=T\left(a_{2}, a_{1}, a_{3}, \ldots, a_{n}\right)=T\left(a_{2}, a_{3}, a_{1}, \ldots, a_{n}\right)$ $=\ldots=T\left(a_{2}, a_{3}, a_{4}, \ldots, a_{n}, a_{1}\right)$,

(iii) $a_{i} \geq b_{i}, i=1,2,3, \ldots, n$ imply $T\left(a_{1}, a_{2}, a_{3}, \ldots, a_{n}\right) \geq T\left(b_{1}, b_{2}, b_{3}, \ldots, b_{n}\right)$,

(iv) $T\left(T\left(a_{1}, a_{2}, a_{3}, \ldots, a_{n}\right), b_{2}, b_{3}, \ldots, b_{n}\right)$

$$
\begin{aligned}
& =T\left(a_{1}, T\left(a_{2}, a_{3}, \ldots, a_{n}, b_{2}\right), b_{3}, \ldots, b_{n}\right) \\
& =T\left(a_{1}, a_{2}, T\left(a_{3}, a_{4}, \ldots, a_{n}, b_{2}, b_{3}\right), b_{4}, \ldots, b_{n}\right) \\
& =\ldots \\
& =T\left(a_{1}, a_{2}, a_{3}, \ldots, a_{n-1}, T\left(a_{n}, b_{2}, b_{3}, \ldots, b_{n}\right)\right) .
\end{aligned}
$$

When $n=2$, we have a binary $t$-norm, which is commonly known as t-norm.

The following are three basic continuous 3-th order $t$-norms:

(1) The minimum 3 -th order $t$-norm, say $T_{M}$, defined by $T_{M}(a, b, c)=\min \{a, b, c\}$.

(2) The product 3 -th order $t$-norm, say $T_{P}$, defined by $T_{P}(a, b, c)=a b c$.

(3) The Lukasiewicz 3 -th order $t$-norm, say $T_{L}$, defined by $T_{L}(a, b, c)=\max \{a+b+c-2,0\}$.

These $t$-norms are related in the following way: $T_{L} \leq T_{P} \leq T_{M}$.

Definition 1.4. [21] A function $F: \mathbb{R} \rightarrow \mathbb{R}^{+}$is called a distribution if it is increasing left-continuous with $\inf _{t \in \mathbb{R}} F(t)=0$ and $\sup _{t \in \mathbb{R}} F(t)=1$.

The set of all distribution functions is denoted by $\mathcal{D}^{+}$. A special distribution function is given by

$$
H(t)= \begin{cases}0, & t \leq 0, \\ 1, & t>0 .\end{cases}
$$

Definition 1.5. [21] A Menger probabilistic metric space (briefly, Menger PM-space) is a triple (X,F,T) where $X$ is a nonempty set, $T$ is a continuous t-norm, and $F$ is a mapping from $X \times X$ into $\mathcal{D}^{+}$such that, if $F_{x, y}$ denotes the value of $F$ at the pair $(x, y)$, the following conditions hold:

(PM1) $F_{x, y}(t)=H(t)$ if and only if $x=y$,

$(\mathrm{PM} 2) F_{x, y}(t)=F_{y, x}(t)$,

(PM3) $F_{x, y}(t+s) \geq T\left(F_{x, z}(t), F_{z, y}(s)\right)$ for all $x, y, z \in X$ and $s, t \geq 0$. 
According to [21], the $(\varepsilon, \lambda)$-topology in a Menger PM-space $(X, F, T)$ is a family of neighborhoods $N_{x}$ of a point $x \in X$ given by $N_{x}=\left\{N_{x}(\varepsilon, \lambda): \varepsilon>0, \lambda \in(0,1)\right\}$, where $N_{x}(\varepsilon, \lambda)=\left\{y \in X: F_{x, y}(\varepsilon)>1-\lambda\right\}$. The $(\varepsilon, \lambda)$-topology is a Hausdorff topology.

Definition 1.6. [21] Let $(X, F, T)$ be a Menger PM-space. Then:

(i) A sequence $\left\{x_{n}\right\}$ in $X$ is said to be convergent to $x$ in $X$ if, for every $\varepsilon>0$ and $\lambda>0$, a positive integer $N$ exists such that for each $n \geq N, F_{x_{n}, x}(\varepsilon)>1-\lambda$.

(ii) A sequence $\left\{x_{n}\right\}$ in $X$ is called a Cauchy sequence if, for every $\varepsilon>0$ and $\lambda>0$, a positive integer $N$ exists such that for each $n, m \geq N, F_{x_{n}, x_{m}}(\varepsilon)>1-\lambda$.

(iii) A Menger PM-space is said to be complete if every Cauchy sequence in $X$ is convergent to a point in $X$.

Example 1.1. Let $X=\mathbb{R}^{+}, T(a, b)=\frac{a b}{a+b-a b}$ if $a, b \in(0,1]$ and $T(a, b)=0$, if $a=0$ or $b=0$. Define $F: X \times X \rightarrow \mathcal{D}^{+}$by

$$
F_{x, y}(t)= \begin{cases}\frac{t}{t+|x-y|}, & \text { if } \quad t>0, \\ 0, & \text { if } \quad t \leq 0\end{cases}
$$

for all $x, y \in X$. Then $(X, F, T)$ is a complete Menger PM-space.

Definition 1.7. [10] A function $\phi: \mathbb{R}^{+} \rightarrow \mathbb{R}^{+}$is said to be a $\Phi$-function if it satisfies the following conditions:

(i) $\phi(t)=0$ if and only if $t=0$,

(ii) $\phi(t)$ is strictly monotone increasing and $\phi(t) \rightarrow \infty$ as $t \rightarrow \infty$,

(iii) $\phi$ is left-continuous in $(0, \infty)$,

(iv) $\phi$ is continuous at 0 .

From now on, we mean by $\Phi$ the class of all $\phi$-functions and by $\Psi$ the class of continuous functions $\psi: \mathbb{R}^{+} \rightarrow \mathbb{R}^{+}$such that $\psi(0)=0$ and $\psi^{n}\left(a_{n}\right) \rightarrow 0$, whenever $a_{n} \rightarrow 0$ as $n \rightarrow \infty$.

\section{MAIN RESULT}

In this section, first we describe the new concept of Menger probabilistic partial metric space. Then we improve some fixed point results of Gopal et al. [10], in the setup of Menger probabilistic partial metric spaces.

Definition 2.1. A probabilistic partial metric space is an ordered pair $(X, F)$ where $X$ is a nonempty set, $F: X \times X \rightarrow \mathcal{D}^{+}$is given by $(x, y) \mapsto F_{x, y}$, such that the following conditions are satisfied for all $x, y, z \in X$ and $t \in \mathbb{R}^{+}$: 
(PPM1) $F_{x, y}(t)=F_{x, x}(t)=F_{y, y}(t)$ if and only if $x=y$,

$(\mathrm{PPM} 2) F_{x, y}(t)=F_{y, x}(t)$,

(PPM3) $F_{x, x}(t) \geq F_{x, y}(t)$,

(PPM4) If $F_{x, z}\left(t_{1}\right)=1, F_{z, y}\left(t_{2}\right)=1$ and $F_{z, z}\left(t_{3}\right)=1$ for $t_{1}, t_{2}, t_{3} \in \mathbb{R}^{+}, \quad$ then $F_{x, y}\left(t_{1}+t_{2}+t_{3}\right)=1$.

It is clear that every probabilistic metric space is a probabilistic partial metric space. However, the converse of this fact needs not hold. For example, $x=y$ does not imply $F_{x, y}(t)=H(t)$. See the following example.

Example 2.1. Let $(X, p)$ be a partial metric space. If $F: X \times X \rightarrow \mathcal{D}^{+}$is a mapping defined by

$$
F_{x, y}(t)=H(t-p(x, y)), \quad \forall x, y \in X, t \in \mathbb{R}
$$

then $(X, F)$ is a probabilistic partial metric space. Obviously, $x=y$ does not imply $F_{x, y}(t)=H(t)$.

Definition 2.2. A Menger probabilistic partial metric space is a triple $(X, F, T)$, where $(X, F)$ is a probabilistic partial metric space, $T$ is a continuous 3-th order t-norm and the following inequality holds:

$$
F_{x, y}\left(t_{1}+t_{2}+t_{3}\right) \geq T\left(F_{x, z}\left(t_{1}\right), F_{z, y}\left(t_{2}\right), F_{z, z}\left(t_{3}\right)\right)
$$

for all $x, y, z \in X$ and all $t_{1}, t_{2}, t_{3} \in \mathbb{R}^{+}$.

Remark 2.1. Let $(X, F)$ be as Example 2.1. Then $\left(X, F, T_{M}\right)$ is a Menger probabilistic partial metric space induced by $(X, p)$.

Example 2.2. Let $(X, p)$ be a partial metric space. Define a mapping $F: X \times X \rightarrow \mathcal{D}^{+}$by

$$
F_{x, y}(t)= \begin{cases}\frac{t}{t+p(x, y)}, & \text { if } \quad t>0 \\ 0, & \text { if } \quad t \leq 0\end{cases}
$$

for all $x, y \in X$. Then $\left(X, F, T_{M}\right)$ is a Menger probabilistic partial metric space.

Definition 2.3. Let $(X, F, T)$ be a Menger probabilistic partial metric space. Then:

(i) A sequence $\left\{x_{n}\right\}$ in $X$ is said to be convergent to $x$ in $X$ if, for each $t>0, \lim _{n \rightarrow \infty} F_{x_{n}, x}(t)=F_{x, x}(t)$.

(ii) $A$ sequence $\left\{x_{n}\right\}$ in $X$ is called a Cauchy sequence if, for each $t>0$, $\lim _{m, n \rightarrow \infty} F_{x_{m}, x_{n}}(t)$ exists.

(iii) A Menger probabilistic partial metric space is said to be complete if for every Cauchy sequence $\left\{x_{n}\right\}$ in $X$, a point $x \in X$ exists such that $\lim _{m, n \rightarrow \infty} F_{x_{m}, x_{n}}(t)=\lim _{n \rightarrow \infty} F_{x_{n}, x}(t)=F_{x, x}(t)$.

(iv) A sequence $\left\{x_{n}\right\}$ is called $G$-Cauchy if for each $p \in \mathbb{N}$ and $t>0, \lim _{n \rightarrow \infty} F_{x_{n}, x_{n+p}}(t)$ exists. 
(v) The space $(X, F, T)$ is called $G$-complete if for every $G$-Cauchy sequence $\left\{x_{n}\right\}$ in $X$, a point $x \in X$ exists such that $\lim _{n \rightarrow \infty} F_{x_{n}, x_{n+p}}(t)=\lim _{n \rightarrow \infty} F_{x_{n}, x}(t)=F_{x, x}(t)$.

Definition 2.4. (see also [10]) Let $X$ be a nonempty set, $f: X \rightarrow X$ be a mapping and $\beta, \gamma: X \times X \times$ $(0, \infty) \rightarrow(0, \infty)$ be two functions. Then $f$ is said to be $(\beta, \gamma)$-admissible if for all $x, y \in X$ and all $t>0$ we have

$$
\beta(x, y, t) \leq 1 \quad \text { implies } \quad \beta(f x, f y, t) \leq 1
$$

and

$$
\gamma(x, y, t) \geq 1 \quad \text { implies } \quad \gamma(f x, f y, t) \geq 1
$$

Definition 2.5. Let $(X, F, T)$ be a Menger probabilistic partial metric space, $f: X \rightarrow X$ be a given mapping and $\beta, \gamma: X \times X \times(0, \infty) \rightarrow(0, \infty)$ be two functions. We say that $f$ is a $(\beta, \gamma)$-admissible $\psi$-type contractive mapping if $f$ is a $(\beta, \gamma)$-admissible mapping, satisfying in the following inequality

$$
\gamma(f x, f y, t)\left(\frac{1}{F_{f x, f y}(\phi(c t))}-1\right) \leq \beta(x, y, t) \psi\left(\frac{1}{F_{x, y}(\phi(t))}-1\right)
$$

for all $x, y \in X$ and all $t>0$ such that $F_{x, y}(\phi(t))>0$, where $c \in(0,1), \phi \in \Phi$ and $\psi \in \Psi$.

According to Gopal et al. [10, Theorem 2.1], we present a new fixed point theorem in the Menger probabilistic partial metric spaces.

Theorem 2.1. Let $(X, F, T)$ be a G-complete Menger probabilistic partial metric space and $f: X \rightarrow X$ be $a(\beta, \gamma)$-admissible $\psi$-type contractive mapping satisfying the following conditions:

(i) $x_{0} \in X$ exists such that $\beta\left(x_{0}, f x_{0}, t\right) \leq 1$ and $\gamma\left(x_{0}, f x_{0}, t\right) \geq 1$ for all $t>0$,

(ii) if $\left\{x_{n}\right\}$ is a sequence in $X$ such that $\beta\left(x_{n-1}, x_{n}, t\right) \leq 1$ and $\gamma\left(x_{n}, x_{n+1}, t\right) \geq 1$ for all $n \in \mathbb{N}$, and for all $t>0$ and $x_{n} \rightarrow x$ as $n \rightarrow \infty$, then $\beta\left(x_{n}, x, t\right) \leq 1$ and $\gamma\left(x_{n}, f x, t\right) \geq 1$ for all $n \in \mathbb{N}$ and for all $t>0$.

Then $f$ has a fixed point.

Proof. Let $x_{0} \in X$ be such that condition (i) holds. We define inductively the sequence $\left\{x_{n}\right\}$ in $X$ by $x_{n+1}=f x_{n}$, for $n=0,1,2, \ldots$. We may suppose that $x_{n+1} \neq x_{n}$ for each $n$, otherwise $f$ has obviously a fixed point. We conclude from $(\beta, \gamma)$-admissibility of the mapping $f$, the condition $(i)$, and by induction that $\beta\left(x_{n-1}, x_{n}, t\right) \leq 1$ and $\gamma\left(x_{n}, x_{n+1}, t\right) \geq 1$ for all $n \in \mathbb{N}$ and all $t>0$. From properties of the function $\phi$, it is possible to find some $t>0$ such that $F_{x_{0}, x_{1}}(\phi(t))>0$. It implies that $F_{x_{0}, x_{1}}\left(\phi\left(\frac{t}{c}\right)\right)>0$, too. From (2.2), we have that

$$
\begin{aligned}
\frac{1}{F_{x_{1}, x_{2}}(\phi(c t))}-1 & \leq \gamma\left(f x_{0}, f x_{1}, t\right)\left(\frac{1}{F_{f x_{0}, f x_{1}}(\phi(c t))}-1\right) \\
& \leq \beta\left(x_{0}, x_{1}, t\right) \psi\left(\frac{1}{F_{x_{0}, x_{1}}(\phi(t))}-1\right) \\
& \leq \psi\left(\frac{1}{F_{x_{0}, x_{1}}(\phi(t))}-1\right)
\end{aligned}
$$


Repeating the above procedure successively $r$ times $(r<n)$, we obtain

$$
\frac{1}{F_{x_{n}, x_{n+1}}\left(\phi\left(c^{r} t\right)\right)}-1 \leq \psi^{n-r}\left(\frac{1}{F_{x_{r}, x_{r+1}}\left(\phi\left(\frac{c^{r} t}{c^{n-r}}\right)\right)}-1\right) \text {. }
$$

Since $\psi^{n}\left(a_{n}\right) \rightarrow 0$, whenever $a_{n} \rightarrow 0$, then from (2.3), for any positive real number $r$ we have

$$
F_{x_{n}, x_{n+1}}\left(\phi\left(c^{r} t\right)\right) \rightarrow 1, \text { as } n \rightarrow \infty
$$

Now, let $\varepsilon>0$ be given and choose $r>0$ so that $\phi\left(c^{r} t\right)<\varepsilon$. Regarding (2.4) we deduce

$$
F_{x_{n}, x_{n+1}}(\varepsilon) \rightarrow 1, \quad \text { as } n \rightarrow \infty, \quad \text { for every } \varepsilon>0
$$

On the other hand, we can write

$$
\begin{aligned}
F_{x_{n}, x_{n+p}}(\varepsilon) & \geq T\left(F_{x_{n}, x_{n+1}}\left(\frac{\varepsilon}{3}\right), F_{x_{n+1}, x_{n+1}}\left(\frac{\varepsilon}{3}\right), F_{x_{n+1}, x_{n+p}}\left(\frac{\varepsilon}{3}\right)\right) \\
& \geq \cdots \\
& \geq T\left(F_{x_{n}, x_{n+1}}\left(\frac{\varepsilon}{3}\right), F_{x_{n}, x_{n+1}}\left(\frac{\varepsilon}{3}\right), T\left(F_{x_{n+1}, x_{n+2}}\left(\frac{\varepsilon}{9}\right), F_{x_{n+1}, x_{n+2}}\left(\frac{\varepsilon}{9}\right),\right.\right. \\
T(\ldots, T & \left.\left.\left(F_{x_{n+p-2}, x_{n+p-1}}\left(\frac{\varepsilon}{3^{p-1}}\right), F_{x_{n+p-2}, x_{n+p-1}}\left(\frac{\varepsilon}{3^{p-1}}\right), F_{x_{n+p-1}, x_{n+p}}\left(\frac{\varepsilon}{3^{p-1}}\right)\right)\right)\right) .
\end{aligned}
$$

On making $n \rightarrow \infty$ and in view of (2.5), for any positive integer $p$, we have

$$
F_{x_{n}, x_{n+p}}(\varepsilon) \rightarrow 1, \quad \text { as } \quad n \rightarrow \infty, \quad \text { for every } \quad \varepsilon>0
$$

It follows that $\left\{x_{n}\right\}$ is a G-Cauchy sequence. Since $(X, F, T)$ is G-complete, $\left\{x_{n}\right\}$ is convergent and

$$
\lim _{n \rightarrow \infty} F_{x_{n}, x_{n+p}}(t)=\lim _{n \rightarrow \infty} F_{x_{n}, u}(t)=F_{u, u}(t)
$$

for some $u \in X$. Furthermore, we get

$$
F_{f u, u}(\varepsilon) \geq T\left(F_{f u, x_{n+1}}\left(\frac{\varepsilon}{3}\right), F_{u, x_{n+1}}\left(\frac{\varepsilon}{3}\right), F_{x_{n+1}, x_{n+1}}\left(\frac{\varepsilon}{3}\right)\right) .
$$

Taking into account the continuity of $\phi$ at zero, $t_{1}>0$ exists such that $\phi\left(t_{1}\right)<\frac{\varepsilon}{3}$. From $(2.6), n_{0} \in \mathbb{N}$ exists such that $F_{x_{n}, u}\left(\phi\left(t_{1}\right)\right)>0$ for all $n \geq n_{0}$. Hence, applying $(i i)$ we derive that

$$
\frac{1}{F_{x_{n+1}, f u}\left(\frac{\varepsilon}{3}\right)}-1 \leq \gamma\left(f x_{n}, f u, t_{1}\right)\left(\frac{1}{F_{f x_{n}, f u}\left(\phi\left(t_{1}\right)\right)}-1\right) \leq \beta\left(x_{n}, u, t_{1}\right) \psi\left(\frac{1}{F_{x_{n}, u}\left(\phi\left(\frac{t_{1}}{c}\right)\right)}-1\right)
$$

for all $n \geq n_{0}$. Now, letting $n \rightarrow \infty$, since $\psi(0)=0$ and by the continuity of function $\psi$, we obtain

$$
F_{x_{n+1}, f u}\left(\frac{\varepsilon}{3}\right) \rightarrow 1, \quad \text { as } \quad n \rightarrow \infty
$$

From (2.1) and (2.6) $F_{x_{n+1}, x_{n+1}}\left(\frac{\varepsilon}{3}\right) \rightarrow 1$ as $n \rightarrow \infty$, too. Passing $n$ to infinity in the relation (2.7) it follows that $F_{f u, u}(\varepsilon)=1$, for each $\varepsilon>0$. Thus $f u=u$. This completes the proof. 
Example 2.3. Let $X=\mathbb{R}^{+}$. Define $F: X \times X \rightarrow \mathcal{D}^{+}$by

$$
F_{x, y}(t)=\frac{t}{t+\max \{x, y\}}
$$

for all $x, y \in X$ and for all $t>0$. Define the mapping $f: X \rightarrow X$ by

$$
f x= \begin{cases}\frac{x}{2}, & \text { if } \quad x \in[0,1), \\ 2 x, & \text { otherwise }\end{cases}
$$

and the functions $\beta$ and $\gamma$ from $X \times X \times(0, \infty)$ into $(0, \infty)$ by

$$
\begin{gathered}
\beta(x, y, t)=\frac{t}{t+|x-y|}, \\
\gamma(x, y, t)= \begin{cases}\frac{t}{t+2(x+y)}, & \text { if } x, y \in[0,1), \\
\frac{1}{4}\left(\frac{t}{t+x+y}\right), & \text { otherwise }\end{cases}
\end{gathered}
$$

for all $t>0$. Now, consider self-mappings $\phi$ and $\psi$ on $\mathbb{R}^{+}$defined by $\phi(t)=\psi(t)=t$ and let $c=\frac{1}{2}$. Obviously, $f$ is $(\beta, \gamma)$-admissible. To show that $f$ is a $(\beta, \gamma)$-admissible $\psi$-type contractive mapping, we have to check the condition (2.2).

To do this, we distinguish three cases:

case I: If $0 \leq x \leq y<1$, then

$$
\begin{aligned}
\gamma(f x, f y, t)\left(\frac{1}{F_{f x, f y}(\phi(c t))}-1\right) & =\frac{y}{t+|x+y|} \\
& \leq \beta(x, y, t) \psi\left(\frac{1}{F_{x, y}(\phi(t))}-1\right) \\
& =\frac{y}{t+|x-y|} .
\end{aligned}
$$

case II: If $x \in[0,1)$ and $y \notin[0,1]$, then

$$
\gamma(f x, f y, t)\left(\frac{1}{F_{f x, f y}(\phi(c t))}-1\right)=\frac{2 y}{2 t+|x+4 y|} \leq \beta(x, y, t) \psi\left(\frac{1}{F_{x, y}(\phi(t))}-1\right)=\frac{y}{t+|x-y|} .
$$

case III: If $x, y \notin[0,1)$, then

$$
\gamma(f x, f y, t)\left(\frac{1}{F_{f x, f y}(\phi(c t))}-1\right)=\frac{y}{t+|2 x+2 y|} \leq \beta(x, y, t) \psi\left(\frac{1}{F_{x, y}(\phi(t))}-1\right)=\frac{y}{t+|x-y|} .
$$

It can be easily verified that all conditions of Theorem 2.1 hold, and therefore $f$ has a fixed point.

We denote by Fix $(f)$ the set of fixed points of $f$. In what follows, we give a sufficient condition for the uniqueness of the fixed point in Theorem 2.1.

$(\mathrm{H})$ : For all $u, v \in$ Fix $(f)$ and for all $t>0$ there exists $z \in X$ such that

$$
\beta(z, f z, t) \leq 1 \quad \text { with } \quad \beta(u, z, t) \leq 1 \quad \text { and } \quad \beta(v, z, t) \leq 1,
$$


and

$$
\gamma(z, f z, t) \geq 1 \quad \text { with } \quad \gamma(u, z, t) \geq 1 \quad \text { and } \quad \gamma(v, z, t) \geq 1
$$

Similar to this condition was already considered in the paper [10].

Theorem 2.2. Adding condition (H) to the hypotheses of Theorem 2.1, we obtain that $f$ has a unique fixed point.

Proof. Let $u, v \in$ Fix $(f)$. From condition $(\mathrm{H}), z \in X$ exists such that

$$
\beta(z, f z, t) \leq 1 \quad \text { with } \quad \beta(u, z, t) \leq 1 \quad \text { and } \quad \beta(v, z, t) \leq 1,
$$

and

$$
\gamma(z, f z, t) \geq 1 \quad \text { with } \quad \gamma(u, z, t) \geq 1 \quad \text { and } \quad \gamma(v, z, t) \geq 1 .
$$

Taking into account $(\beta, \gamma)$-admissibility of $f$, we obtain

$$
\beta\left(f z, f^{2} z, t\right) \leq 1, \quad \beta(u, f z, t) \leq 1, \quad \beta(v, f z, t) \leq 1
$$

and

$$
\gamma\left(f z, f^{2} z, t\right) \geq 1, \quad \gamma(u, f z, t) \geq 1, \quad \gamma(v, f z, t) \geq 1 .
$$

Consequently, by induction, we get

$$
\beta\left(z_{n}, z_{n+1}, t\right) \leq 1, \quad \beta\left(u, z_{n}, t\right) \leq 1, \quad \beta\left(v, z_{n}, t\right) \leq 1
$$

and

$$
\gamma\left(z_{n+1}, z_{n+2}, t\right) \geq 1, \quad \gamma\left(u, z_{n+1}, t\right) \geq 1, \quad \gamma\left(v, z_{n+1}, t\right) \geq 1
$$

for all $t>0$, where $z_{n}=f^{n} z$. Then, using (2.2) we derive

$$
\begin{aligned}
\frac{1}{F_{u, f^{n} z}(\phi(c t))}-1 & \leq \gamma\left(u, z_{n}, t\right)\left(\frac{1}{F_{f u, f\left(f^{n-1} z\right)}(\phi(c t))}-1\right) \\
& \leq \beta\left(u, z_{n-1}, t\right) \psi\left(\frac{1}{F_{u, f^{n-1} z}(\phi(t))}-1\right) \\
& \leq \psi\left(\frac{1}{F_{u, f^{n-1} z}(\phi(t))}-1\right) .
\end{aligned}
$$

It follows that

$$
\frac{1}{F_{u, f^{n} z}(\phi(c t))}-1 \leq \psi^{n}\left(\frac{1}{F_{u, z}\left(\phi\left(\frac{t}{c^{n-1}}\right)\right)}-1\right) \quad \text { for all } n \in \mathbb{N} .
$$

Letting $n \rightarrow \infty$, we obtain $\lim _{n \rightarrow \infty} F_{u, z_{n+1}}=1$. A similar argument shows that $\lim _{n \rightarrow \infty} F_{v, z_{n+1}}=1$. Using these facts, it is easily can be shown that $F_{u, v}(t)=1$ for any $t>0$. It implies that $u=v$, and the proof is complete. 
Example 2.4. Let $X=\mathbb{R}^{+}$and $F_{x, y}(t)=H(t-\max \{x, y\})$ for all $x, y \in X$ and for all $t>0$. Clearly, $\left(X, F, T_{p}\right)$ is a G-complete Menger probabilistic partial metric space. Define the mapping $f: X \rightarrow X$ by

$$
f(x)=\frac{x}{2}
$$

and the functions $\gamma$ and $\beta$ from $X \times X \times(0, \infty)$ into $(0, \infty)$ by

$$
\begin{gathered}
\beta(x, y, t)= \begin{cases}2, & \text { if } x, y \in(2, \infty), \\
\frac{t}{t+|x-y|}, & \text { otherwise }\end{cases} \\
\gamma(x, y, t)= \begin{cases}\frac{1}{3}, & \text { if } x, y \in(5, \infty), \\
5, & \text { otherwise }\end{cases}
\end{gathered}
$$

for all $t>0$. Also suppose that $\phi, \psi: \mathbb{R}^{+} \rightarrow \mathbb{R}^{+}$defined by $\phi(t)=\psi(t)=t$ and let $c=\frac{1}{2}$. Now, it can be easily shown that all the hypotheses of Theorem 2.2 are satisfied and so $f$ has a unique fixed point.

In the sequel, we first introduce the concept of $(\beta, \gamma)$-contractive mapping of type (I) and then we describe a fixed point theorem concerned with these kinds of contractions in the framework of Menger probabilistic partial metric spaces.

Definition 2.6. Let $(X, F, T)$ be a Menger probabilistic partial metric space and $f: X \rightarrow X$ be a given mapping. We say that $f$ is a $(\beta, \gamma)$-contractive mapping of type (I), if functions $\beta: X \times X \times(0, \infty) \rightarrow(0, \infty)$ and $\gamma: X \times X \times(0, \infty) \rightarrow(0, \infty)$ exist such that

$$
\beta(x, y, t) F_{f x, f y}(\phi(t)) \geq \gamma\left(f x, f y, \frac{t}{c}\right) \min \left\{F_{x, y}\left(\phi\left(\frac{t}{c}\right)\right), F_{y, f y}\left(\phi\left(\frac{t}{c}\right)\right), F_{y, f x}\left(\phi\left(\frac{t}{c}\right)\right)\right\}
$$

for all $x, y \in X$ and all $t>0$, where $\phi \in \Phi$ and $c \in(0,1)$.

Example 2.5. Let $X=\{0,2,4\}$ and $F$ be as in Example 2.3. Clearly, $\left(X, F, T_{M}\right)$ is a complete Menger probabilistic partial metric space. Define the mapping $f: X \rightarrow X$ by $f(0)=0, f(2)=4$ and $f(4)=2$. Also, define two functions $\beta$ and $\gamma$ from $X \times X \times(0, \infty)$ into $(0, \infty)$ by

$$
\begin{gathered}
\beta(x, y, t)= \begin{cases}2, & \text { if } \quad x=y, \\
\frac{1}{2}, & \text { otherwise }\end{cases} \\
\gamma(x, y, t)= \begin{cases}1, & \text { if } x=y=2, \text { or } x=y=4, \\
2, & \text { if } x=y=0, \\
\frac{1}{x+y+1}, & \text { otherwise. }\end{cases}
\end{gathered}
$$

Now, consider $\phi: \mathbb{R}^{+} \rightarrow \mathbb{R}^{+}$defined by $\phi(t)=t$ and let $c=\frac{1}{2}$. Then $f$ is a $(\beta, \gamma)$-contractive mapping of type (I). 
Two useful following lemmas help us to prove Theorem 2.3.

Lemma 2.1. Let $(X, F, T)$ be a Menger probabilistic partial metric space and $\phi$ be a $\Phi$-function. Then the following statement holds.

If for $x, y \in X$ and $c \in(0,1)$ we have $F_{x, y}(\phi(t)) \geq F_{x, y}\left(\phi\left(\frac{t}{c}\right)\right)$ for all $t>0$, then $x=y$.

Proof. The proof is similar to [4, Lemma 2.9].

Lemma 2.2. Let $(X, F, T)$ be a Menger probabilistic partial metric space. Then the function $F$ is a lower semi-continuous function of points, i.e., for every fixed $t>0$ and every two sequences $\left\{x_{n}\right\},\left\{y_{n}\right\}$ in $X$ such that $\lim _{n \rightarrow \infty} F_{x_{n}, x}(t)=F_{x, x}(t)=1$ and $\lim _{n \rightarrow \infty} F_{y_{n}, y}(t)=F_{y, y}(t)=1$ it follows that $\liminf _{n \rightarrow \infty} F_{x_{n}, y_{n}}(t)=F_{x, y}(t)$.

Proof. Let $t>0$ and $\varepsilon>0$ be given. Since $F_{x, y}$ is left-continuous at $t$, so $h$ exists such that $0<2 h<t$ and $F_{x, y}(t)-F_{x, y}(t-2 h)<\varepsilon$. Put $F_{x, y}(t-2 h)=a$. Taking into account continuity of $T$ and $T(a, 1,1)=a$, there is a real number $l$ in $(0,1)$, fulfills

$$
T(a, l, l)>a-\frac{\varepsilon}{3} \quad \text { and } \quad T\left(a-\frac{\varepsilon}{3}, l, l\right)>a-\frac{2 \varepsilon}{3} .
$$

On the other hand, by our assumptions, an integer $M_{h, l}$ exists such that

$$
F_{x_{n}, x}\left(\frac{h}{2}\right)>l \quad \text { and } \quad F_{y_{n}, y}\left(\frac{h}{2}\right)>l
$$

whenever $n>M_{h, l}$. Now, by (2.1) and (PPM3)

$$
F_{x_{n}, y_{n}}(t) \geq T\left(F_{x_{n}, y}(t-h), F_{y, y_{n}}\left(\frac{h}{2}\right), F_{y, y_{n}}\left(\frac{h}{2}\right)\right)
$$

and

$$
F_{x_{n}, y}(t-h) \geq T\left(F_{x_{n}, x}\left(\frac{h}{2}\right), F_{x, y}(t-2 h), F_{x, x}\left(\frac{h}{2}\right)\right)>T(a, l, l)>a-\frac{\varepsilon}{3} .
$$

Thus, on combining (2.9) and (2.10), we have

$$
F_{x_{n}, y_{n}}(t) \geq T\left(a-\frac{\varepsilon}{3}, l, l\right)>a-\frac{2 \varepsilon}{3}>F_{x, y}(t)-\varepsilon .
$$

This completes the proof.

Now, we present a new version of [10, Theorem 3.2] due to Gopal et al. in the Menger probabilistic partial metric spaces.

Theorem 2.3. Let $(X, F, T)$ be a complete Menger probabilistic partial metric space, which satisfies $T(a, a, a) \geq$ a with $a \in[0,1]$. Let $f: X \rightarrow X$ be a $(\beta, \gamma)$-contractive mapping of type (I) satisfying the following conditions:

(i) $f$ is $(\beta, \gamma)$-admissible,

(ii) there exists $x_{0} \in X$ such that $\beta\left(x_{0}, f x_{0}, t\right) \leq 1$ and $\gamma\left(x_{0}, f x_{0}, t\right) \geq 1$ for all $t>0$, 
(iii) if $\left\{x_{n}\right\}$ is a sequence in $X$ such that $\beta\left(x_{n-1}, x_{n}, t\right) \leq 1$ and $\gamma\left(x_{n}, x_{n+1}, t\right) \geq 1$ for all $n \in \mathbb{N}$, and all $t>0$ and $x_{n} \rightarrow x$ as $n \rightarrow \infty$, then $\beta\left(x_{n-1}, x, t\right) \leq 1$ and $\gamma\left(x_{n}, f x, t\right) \geq 1$ for all $n \in \mathbb{N}$ and all $t>0$.

Then $f$ has a fixed point.

Proof. Since $T(a, a, a) \geq a$ for all $a \in[0,1]$, then $T \geq T_{M}$. Let $x_{0} \in X$ be such that (ii) holds and define a sequence $\left\{x_{n}\right\}$ in $X$ so that $x_{n+1}=f x_{n}$, for $n=0,1, \ldots$ We suppose $x_{n+1} \neq x_{n}$ for all $n=0,1, \ldots$, otherwise $f$ has trivially a fixed point. From $(i),(i i)$ and by induction, we get $\beta\left(x_{n-1}, x_{n}, t\right) \leq 1$ and $\gamma\left(x_{n}, x_{n+1}, t\right) \geq 1$ for all $n \in \mathbb{N}$ and all $t>0$. By continuity of $\phi$ at zero, for each $t>0$ we can find $r>0$ such that $t>\phi(r)$ and therefore using (2.8) and (PPM3) we have

$$
\begin{aligned}
F_{x_{n}, x_{n+1}}(t) & \geq \beta\left(x_{n-1}, x_{n}, r\right) F_{f x_{n-1}, f x_{n}}(\phi(r)) \\
& \geq \gamma\left(x_{n}, x_{n+1}, \frac{r}{c}\right) \min \left\{F_{x_{n-1}, x_{n}}\left(\phi\left(\frac{r}{c}\right)\right), F_{x_{n}, f x_{n}}\left(\phi\left(\frac{r}{c}\right)\right), F_{x_{n}, f x_{n-1}}\left(\phi\left(\frac{r}{c}\right)\right)\right\} \\
& \geq \min \left\{F_{x_{n-1}, x_{n}}\left(\phi\left(\frac{r}{c}\right)\right), F_{x_{n}, x_{n+1}}\left(\phi\left(\frac{r}{c}\right)\right)\right\} .
\end{aligned}
$$

We will show that

$$
F_{x_{n}, x_{n+1}}(\phi(r)) \geq F_{x_{n-1}, x_{n}}\left(\phi\left(\frac{r}{c}\right)\right) .
$$

If we assume that $F_{x_{n}, x_{n+1}}\left(\phi\left(\frac{r}{c}\right)\right)$ is the minimum, then from Lemma 2.1, we deduce that $x_{n}=x_{n+1}$. This is in contradiction with the assumption $x_{n} \neq x_{n+1}$ and so $F_{x_{n-1}, x_{n}}\left(\phi\left(\frac{r}{c}\right)\right)$ is the minimum i.e., inequality (2.11) holds. Now, from (2.11), it follows that

$$
F_{x_{n}, x_{n+1}}(t) \geq F_{x_{n}, x_{n+1}}(\phi(r)) \geq F_{x_{n-1}, x_{n}}\left(\phi\left(\frac{r}{c}\right)\right) \geq \ldots \geq F_{x_{0}, x_{1}}\left(\phi\left(\frac{r}{c^{n}}\right)\right),
$$

that is,

$$
F_{x_{n}, x_{n+1}}(t) \geq F_{x_{0}, x_{1}}\left(\phi\left(\frac{r}{c^{n}}\right)\right),
$$

for arbitrary $n \in \mathbb{N}$. Next, let $m, n \in \mathbb{N}$ with $m>n$, then by (2.1) and (PPM3) we get

$$
F_{x_{n}, x_{m}}((2(m-n)-1) t) \geq T\left(F_{x_{n}, x_{n+1}}(t), F_{x_{n}, x_{n+1}}(t), F_{x_{n+1}, x_{m}}((2(m-n)-3) t)\right) .
$$

By strictly increasing of $\phi$ and also making use of (2.12) we have

$$
\begin{aligned}
F_{x_{n}, x_{m}}((2(m-n)-1) t) & \geq \min \left\{F_{x_{n}, x_{n+1}}(t), \ldots, F_{x_{m-1}, x_{m-2}}(t), F_{x_{m-1}, x_{m}}(t)\right\} \\
& \geq \min \left\{F_{x_{0}, x_{1}}\left(\phi\left(\frac{r}{c^{n}}\right)\right), \ldots, F_{x_{0}, x_{1}}\left(\phi\left(\frac{r}{c^{m-2}}\right)\right), F_{x_{0}, x_{1}}\left(\phi\left(\frac{r}{c^{m-1}}\right)\right)\right\} \\
& =F_{x_{0}, x_{1}}\left(\phi\left(\frac{r}{c^{n}}\right)\right) .
\end{aligned}
$$

Since $\phi\left(\frac{r}{c^{n}}\right) \rightarrow \infty$ as $n \rightarrow \infty$, for fixed $\varepsilon \in(0,1), n_{0} \in \mathbb{N}$ exists such that for each $n \geq n_{0}, F_{x_{0}, x_{1}}\left(\phi\left(\frac{r}{c^{n}}\right)\right)>$ $1-\varepsilon$. This implies that, for every $m>n \geq n_{0}$,

$$
F_{x_{n}, x_{m}}((2(m-n)-1) t)>1-\varepsilon
$$


By the arbitrariness of $t>0$ and $\varepsilon \in(0,1)$, we deduce that $\left\{x_{n}\right\}$ is a Cauchy sequence in the complete Menger probabilistic partial metric space $(X, F, T)$. Thus, $u \in X$ exists such that

$$
\lim _{m, n \rightarrow \infty} F_{x_{m}, x_{n}}(t)=\lim _{n \rightarrow \infty} F_{x_{n}, u}(t)=F_{u, u}(t)
$$

We are going to show that $u \in F i x(f)$. Indeed, we have

$$
\begin{aligned}
F_{f u, u}(2 t) & \geq T\left(F_{f u, x_{n}}(\phi(r)), F_{x_{n}, u}(2 t-2 \phi(r)), F_{x_{n}, x_{n}}(\phi(r))\right) \\
& \geq \min \left\{F_{f u, x_{n}}(\phi(r)), F_{x_{n}, u}(2 t-2 \phi(r))\right\} .
\end{aligned}
$$

We may assume that $x_{n} \neq f u$ for all $n \in \mathbb{N}$, since otherwise if $x_{n}=f u$ for infinitely many values of $n$, then $u=f u$ and hence the proof is finished. Now, from (2.13), for any arbitrary $\varepsilon \in(0,1)$ and $n$ large enough, we get $F_{x_{n}, u}(2 t-2 \phi(r))>1-\varepsilon$. Hence, $F_{f u, u}(2 t) \geq \min \left\{F_{f u, x_{n}}(\phi(r)), 1-\varepsilon\right\}$. Since $\varepsilon>0$ is arbitrary, it yields that $F_{f u, u}(2 t) \geq F_{f u, x_{n}}(\phi(r))$. Next, using $(2.8)$ we deduce

$$
\begin{aligned}
F_{u, f u}(2 t) & \geq F_{x_{n}, f u}(\phi(r)) \\
& \geq \beta\left(x_{n-1}, u, r\right) F_{f x_{n-1}, f u}(\phi(r)) \\
& \geq \gamma\left(f x_{n-1}, f u, \frac{r}{c}\right) \min \left\{F_{x_{n-1}, u}\left(\phi\left(\frac{r}{c}\right)\right), F_{u, f u}\left(\phi\left(\frac{r}{c}\right)\right), F_{u, f x_{n-1}}\left(\phi\left(\frac{r}{c}\right)\right)\right\} \\
& \geq \min \left\{F_{x_{n-1}, u}\left(\phi\left(\frac{r}{c}\right)\right), F_{u, f u}\left(\phi\left(\frac{r}{c}\right)\right), F_{u, x_{n}}\left(\phi\left(\frac{r}{c}\right)\right)\right\} .
\end{aligned}
$$

By taking the limit infimum on both sides of the above inequality and applying Lemma 2.2, we have

$$
\begin{aligned}
F_{u, f u}(2 t) & \geq \lim _{n \rightarrow \infty} \inf F_{x_{n}, f u}(\phi(r)) \\
& \geq \lim _{n \rightarrow \infty} \inf \min \left\{F_{x_{n-1}, u}\left(\phi\left(\frac{r}{c}\right)\right), F_{u, f u}\left(\phi\left(\frac{r}{c}\right)\right), F_{u, x_{n}}\left(\phi\left(\frac{r}{c}\right)\right)\right\} \\
& \geq \min \left\{1-\varepsilon, F_{u, f u}\left(\phi\left(\frac{r}{c}\right)\right), 1-\varepsilon\right\} .
\end{aligned}
$$

Finally, since $\varepsilon \in(0,1)$ is arbitrary, then $F_{f u, u}(\phi(r)) \geq F_{u, f u}\left(\phi\left(\frac{r}{c}\right)\right)$. From Lemma 2.1, we conclude that $u=f u$ and so we achieve our desired goal.

Example 2.6. Let $X=[0,1], p(x, y)=\max \{x, y\}$ and $(X, F, T)$ be as in Example 2.1. Then $(X, F, T)$ is a complete Menger probabilistic partial metric space. Define the mapping $f: X \rightarrow X$ by

$$
f x= \begin{cases}\frac{x}{4}, & \text { if } \quad x \in\left[0, \frac{1}{2}\right) \cup\left(\frac{1}{2}, 1\right], \\ 0, & \text { if } \quad x=\frac{1}{2},\end{cases}
$$

and the functions $\beta$ and $\gamma$ from $X \times X \times(0, \infty)$ into $(0, \infty)$ by

$$
\beta(x, y, t)=\frac{t+\max \{x, y\}}{t+\min \{x, y\}},
$$




$$
\gamma(x, y, t)=\frac{t}{x+y+t}
$$

We consider $\phi: \mathbb{R}^{+} \rightarrow \mathbb{R}^{+}$defined by $\phi(t)=t$ and let $c=\frac{1}{2}$. It is routine to see that all the hypotheses of Theorem 2.3 are satisfied, and therefore $f$ has a fixed point.

Theorem 2.4. Adding condition $(H)$ to the hypotheses of Theorem 2.3, we obtain that $f$ has a unique fixed point.

Proof. Let $u, v \in X$ be such that $u=f u$ and $v=f v$. From condition $(\mathrm{H}), z \in X$ exists such that $\beta(z, f z, t) \leq 1$ with $\beta(u, z, t) \leq 1$ and $\beta(v, z, t) \leq 1$, and $\gamma(z, f z, t) \geq 1$ with $\gamma(u, z, t) \geq 1$ and $\gamma(v, z, t) \geq 1$. By virtue of the fact that $f$ is $(\beta, \gamma)$-admissible and using induction, we derive

$$
\beta\left(z_{n}, z_{n+1}, t\right) \leq 1, \quad \beta\left(u, z_{n}, t\right) \leq 1, \quad \beta\left(v, z_{n}, t\right) \leq 1
$$

and

$$
\gamma\left(z_{n+1}, z_{n+2}, t\right) \geq 1, \quad \gamma\left(u, z_{n+1}, t\right) \geq 1, \quad \gamma\left(v, z_{n+1}, t\right) \geq 1
$$

for all $t>0$, where $z_{n}=f^{n} z(n \in \mathbb{N})$. By continuity of $\phi, r>0$ exists such that $t>\phi(r)$ and therefore we have

$$
\begin{aligned}
F_{u, z_{n+1}}(t) & \geq F_{u, z_{n+1}}(\phi(r)) \\
& \geq \beta\left(u, z_{n}, r\right) F_{f u, f z_{n}}(\phi(r)) \\
& \geq \gamma\left(f u, f z_{n}, \frac{r}{c}\right) \min \left\{F_{u, z_{n}}\left(\phi\left(\frac{r}{c}\right)\right), F_{z_{n}, z_{n+1}}\left(\phi\left(\frac{r}{c}\right)\right), F_{z_{n}, f u}\left(\phi\left(\frac{r}{c}\right)\right)\right\} \\
& \geq \min \left\{F_{u, z_{n}}\left(\phi\left(\frac{r}{c}\right)\right), F_{z_{n}, z_{n+1}}\left(\phi\left(\frac{r}{c}\right)\right)\right\} .
\end{aligned}
$$

Now, we consider following cases:

case I. If $F_{z_{n}, z_{n+1}}\left(\phi\left(\frac{r}{c}\right)\right)$ is the minimum, then by (2.8) and (PPM3), it follows that

$$
\begin{aligned}
F_{u, z_{n+1}}(\phi(r)) & \geq F_{z_{n}, z_{n+1}}\left(\phi\left(\frac{r}{c}\right)\right) \\
& \geq \beta\left(z_{n-1}, z_{n}, \frac{r}{c}\right) F_{f z_{n-1}, f z_{n}}\left(\phi\left(\frac{r}{c}\right)\right) \\
& \geq \gamma\left(z_{n}, z_{n+1}, \frac{r}{c^{2}}\right) \min \left\{F_{z_{n-1}, z_{n}}\left(\phi\left(\frac{r}{c^{2}}\right)\right), F_{z_{n}, f z_{n}}\left(\phi\left(\frac{r}{c^{2}}\right)\right), F_{z_{n}, z_{n}}\left(\phi\left(\frac{r}{c^{2}}\right)\right)\right\} \\
& \geq \min \left\{F_{z_{n-1}, z_{n}}\left(\phi\left(\frac{r}{c^{2}}\right)\right), F_{z_{n}, z_{n+1}}\left(\phi\left(\frac{r}{c^{2}}\right)\right)\right\} .
\end{aligned}
$$

Now, if $F_{z_{n}, z_{n+1}}\left(\phi\left(\frac{r}{c^{2}}\right)\right)$ is the minimum for some $n \in \mathbb{N}$, then by Lemma 2.1, we deduce that $z_{n}=z_{n+1}$. Applying (PPM3), we get $F_{u, z_{n+1}}(\phi(r)) \geq F_{z_{n}, z_{n+1}}\left(\phi\left(\frac{r}{c^{2}}\right)\right) \geq F_{u, z_{n+1}}\left(\phi\left(\frac{r}{c^{2}}\right)\right)$, then $u=z_{n+1}$. Consequently 
$\beta(v, u, t) \leq 1$ and $\gamma(f v, f u, t) \geq 1$ for all $t>0$ and thus we have

$$
\begin{aligned}
F_{v, u}(\phi(t)) & \geq \beta(v, u, t) F_{f v, f u}(\phi(t)) \\
& \geq \gamma\left(f v, f u, \frac{t}{c}\right) \min \left\{F_{v, u}\left(\phi\left(\frac{t}{c}\right)\right), F_{u, f u}\left(\phi\left(\frac{t}{c}\right)\right), F_{u, f v}\left(\phi\left(\frac{t}{c}\right)\right)\right\} \\
& \geq F_{v, u}\left(\phi\left(\frac{t}{c}\right)\right) .
\end{aligned}
$$

Again, by Lemma 2.1, we have $u=v$.

On the other hand, if $F_{z_{n-1}, z_{n}}\left(\phi\left(\frac{r}{c^{2}}\right)\right)$ is the minimum, then

$$
F_{z_{n}, z_{n+1}}\left(\phi\left(\frac{r}{c}\right)\right) \geq F_{z_{n-1}, z_{n}}\left(\phi\left(\frac{r}{c^{2}}\right)\right) \geq \ldots \geq F_{z_{0}, z_{1}}\left(\phi\left(\frac{r}{c^{n+1}}\right)\right)
$$

and, letting $n \rightarrow \infty$, we get $F_{z_{n}, z_{n+1}}\left(\phi\left(\frac{r}{c}\right)\right) \rightarrow 1$. Therefore $\lim _{n \rightarrow \infty} F_{u, z_{n+1}}(t)=1$. A similar method shows that $\lim _{n \rightarrow \infty} F_{v, z_{n+1}}(t)=1$. By virtue of these facts, we get $F_{u, v}(t)=1$ for each $t>0$. Hence, $u=v$.

case II. Suppose that $F_{u, z_{n}}\left(\phi\left(\frac{r}{c}\right)\right)$ is the minimum, then we get

$$
F_{u, z_{n+1}}(\phi(r)) \geq F_{u, z_{n}}\left(\phi\left(\frac{r}{c}\right)\right) \geq F_{u, z_{n-1}}\left(\phi\left(\frac{r}{c^{2}}\right)\right) \geq \ldots \geq F_{u, z_{0}}\left(\phi\left(\frac{r}{c^{n+1}}\right)\right) .
$$

A similar argument as above shows that $u=v$, and the proof is complete.

In the sequel, we first introduce the concept of $(\beta, \gamma)$-contractive mapping of type (II) and then we describe a fixed point theorem concerned with these kinds of contractions in the setup Menger probabilistic partial metric spaces.

Definition 2.7. Let $(X, F, T)$ be a Menger probabilistic partial metric space and $f: X \rightarrow X$ be a given mapping. We say that $f$ is a $(\beta, \gamma)$-contractive mapping of type (II), if functions $\beta: X \times X \times(0, \infty) \rightarrow(0, \infty)$ and $\gamma: X \times X \times(0, \infty) \rightarrow(0, \infty)$ exist such that

$$
\beta(x, y, t) F_{f x, f y}(\phi(t)) \geq \gamma\left(f x, f y, \frac{t}{c}\right) \min \left\{F_{x, f x}\left(\phi\left(\frac{t}{c}\right)\right), F_{y, f y}\left(\phi\left(\frac{t}{c}\right)\right)\right\}
$$

for all $x, y \in X$, for all $t>0$, where $c \in(0,1)$ and $\phi \in \Phi$.

Now, we present a new version of [10, Theorem 3.4] due to Gopal et al. in the Menger probabilistic partial metric spaces.

Theorem 2.5. Let $(X, F, T)$ be a complete Menger probabilistic partial metric space and $f: X \rightarrow X$ be a $(\beta, \gamma)$-contractive mapping of type (II). Suppose that the following conditions hold:

(i) $f$ is $(\beta, \gamma)$-admissible,

(ii) $x_{0} \in X$ exists such that $\beta\left(x_{0}, f x_{0}, t\right) \leq 1$ and $\gamma\left(x_{0}, f x_{0}, t\right) \geq 1$ for all $t>0$,

(iii) for each sequence $\left\{x_{n}\right\}$ in $X$ such that $\beta\left(x_{n-1}, x_{n}, t\right) \leq 1$ and $\gamma\left(x_{n}, x_{n+1}, t\right) \geq 1$, for all $n \in \mathbb{N}$ and all $t>0, k_{0} \in \mathbb{N}$ exists such that $\beta\left(x_{m-1}, x_{n-1}, t\right) \leq 1$ and $\gamma\left(x_{m}, x_{n}, t\right) \geq 1$, for all $m, n \in \mathbb{N}$ with $m>n \geq k_{0}$ and for all $t>0$, 
(iv) if $\left\{x_{n}\right\}$ is a sequence in $X$ such that $\beta\left(x_{n-1}, x_{n}, t\right) \leq 1$ and $\gamma\left(x_{n}, x_{n+1}, t\right) \geq 1$ for all $n \in \mathbb{N}$ and all $t>0$ and $x_{n} \rightarrow x$ as $n \rightarrow \infty$, then $\beta\left(x_{n-1}, x, t\right) \leq 1$ and $\gamma\left(x_{n}, f x, t\right) \geq 1$ for all $n \in \mathbb{N}$ and all $t>0$.

Then $f$ has a fixed point. In addition, if condition $(H)$ holds, then $f$ has a unique fixed point.

Proof. Let $x_{0} \in X$ be such that (ii) holds. Define a sequence $\left\{x_{n}\right\}$ in $X$ such that $x_{n+1}=f x_{n}$ for all $n=0,1, \ldots$. We may suppose that $x_{n+1} \neq x_{n}$ for all $n=0,1, \ldots$, otherwise $f$ has trivially a fixed point. By $(i)$ and (ii), and applying induction, we get $\beta\left(x_{n-1}, x_{n}, t\right) \leq 1$ and $\gamma\left(x_{n}, x_{n+1}, t\right) \geq 1$ for all $n$ and all $t>0$. By continuity of $\phi$ at zero, for each $t>0, r>0$ exists such that $t>\phi(r)$, thus $\beta\left(x_{n-1}, x_{n}, r\right) \leq 1$ and $\gamma\left(x_{n}, x_{n+1}, \frac{r}{c}\right) \geq 1$. It follows from condition (2.14) and (PPM1) that

$$
\begin{aligned}
\left.F_{x_{n}, x_{n+1}}(t)\right) & \geq F_{f x_{n-1}, f x_{n}}(\phi(r)) \\
& \geq \beta\left(x_{n-1}, x_{n}, r\right) F_{f x_{n-1}, f x_{n}}(\phi(r)) \\
& \geq \gamma\left(x_{n}, x_{n+1}, \frac{r}{c}\right) \min \left\{F_{x_{n-1}, x_{n}}\left(\phi\left(\frac{r}{c}\right)\right), F_{x_{n}, x_{n+1}}\left(\phi\left(\frac{r}{c}\right)\right),\right\} \\
& \geq \min \left\{F_{x_{n-1}, x_{n}}\left(\phi\left(\frac{r}{c}\right)\right), F_{x_{n}, x_{n+1}}\left(\left(\frac{r}{c}\right)\right)\right\} .
\end{aligned}
$$

Now, if $F_{x_{n}, x_{n+1}}\left(\phi\left(\frac{r}{c}\right)\right)$ is the minimum, then $F_{x_{n}, x_{n+1}}(\phi(r)) \geq F_{x_{n}, x_{n+1}}\left(\phi\left(\frac{r}{c}\right)\right)$ and so by Lemma 2.1, $x_{n}=$ $x_{n+1}$, which contradicts the assumption $x_{n} \neq x_{n+1}$. Thus $F_{x_{n-1}, x_{n}}\left(\phi\left(\frac{r}{c}\right)\right)$ is the minimum, and so

$$
F_{x_{n}, x_{n+1}}(t) \geq F_{x_{n-1}, x_{n}}\left(\phi\left(\frac{r}{c}\right)\right) \geq \ldots \geq F_{x_{0}, x_{1}}\left(\phi\left(\frac{r}{c^{n}}\right)\right) .
$$

Letting $n \rightarrow \infty$, then

$$
F_{x_{n}, x_{n+1}}(t) \rightarrow 1
$$

We claim that $\left\{x_{n}\right\}$ is a Cauchy sequence. Suppose the contrary. Then there exist $\varepsilon>0, \lambda \in(0,1)$ for which we can find subsequences $\left\{x_{m(s)}\right\}$ and $\left\{x_{n(s)}\right\}$ of $\left\{x_{n}\right\}$ such that $n(s)$ is the smallest index for which

$$
s<m(s)<n(s), \quad F_{x_{m(s)}, x_{n(s)}}(\varepsilon) \leq 1-\lambda, \quad F_{x_{m(s)}, x_{n(s)-1}}(\varepsilon)>1-\lambda .
$$

By the properties of $\phi, \varepsilon_{1}>0$ exists such that

$$
\phi\left(\varepsilon_{1}\right)<\varepsilon
$$

We deduce that $F_{x_{m(s)}, x_{n(s)}}\left(\phi\left(\varepsilon_{1}\right)\right) \leq 1-\lambda$, so $\left\{x_{n}\right\}$ is not Cauchy sequence with respect to $\phi\left(\varepsilon_{1}\right)$ and $\lambda$. Thus, increasing sequences of integers $m(s)$ and $n(s)$ exist such that $n(s)$ is the smallest index for which

$$
s<m(s)<n(s), \quad F_{x_{m(s)}, x_{n(s)}}\left(\phi\left(\varepsilon_{1}\right)\right) \leq 1-\lambda, \quad F_{x_{m(s)}, x_{n(s)-1}}\left(\phi\left(\varepsilon_{1}\right)\right)>1-\lambda .
$$

Take a real number $\eta$ such that $0<\eta<\phi\left(\frac{\varepsilon_{1}}{c}\right)-\phi\left(\varepsilon_{1}\right)$. From (2.16) it follows that

$$
F_{x_{m(s)}, x_{n(s)-1}}\left(\phi\left(\frac{\varepsilon_{1}}{c}\right)-\eta\right)>1-\lambda .
$$


Then, for any $0<\lambda_{1}<\lambda<1$, by (2.15) it is possible to find a positive integer $N_{1}$ such that for all $s>N_{1}$, we have

$$
F_{x_{m(s)-1}, x_{m(s)}}(\eta)>1-\lambda_{1}, \quad F_{x_{n(s)-1}, x_{n(s)}}(\eta)>1-\lambda_{1} .
$$

From (2.17) it follows that

$$
F_{x_{m(s)-1}, x_{m(s)}}\left(\phi\left(\frac{\varepsilon_{1}}{c}\right)\right) \geq F_{x_{m(s)-1}, x_{m(s)}}(\eta)>1-\lambda_{1}>1-\lambda
$$

A similar relation holds when one substitutes $x_{m(s)-1}$ and $x_{m(s)}$ with $x_{n(s)-1}$ and $x_{n(s)}$, respectively. On the other hand, from (2.18) we observe that

$$
\begin{aligned}
1-\lambda & \geq F_{x_{m(s)}, x_{n(s)}}\left(\phi\left(\varepsilon_{1}\right)\right)=F_{f x_{m(s)-1}, f x_{n(s)-1}}\left(\phi\left(\varepsilon_{1}\right)\right) \\
& \geq \beta\left(x_{m(s)-1}, x_{n(s)-1}, \varepsilon_{1}\right) F_{f x_{m(s)-1}, f x_{n(s)-1}}\left(\phi\left(\varepsilon_{1}\right)\right) \\
& \geq \gamma\left(f x_{m(s)-1}, f x_{n(s)-1}, \frac{\varepsilon_{1}}{c}\right) \min \left\{F_{x_{m(s)-1}, x_{m(s)}}\left(\phi\left(\frac{\varepsilon_{1}}{c}\right)\right) F_{x_{n(s)-1}, x_{n(s)}}\left(\phi\left(\frac{\varepsilon_{1}}{c}\right)\right)\right\} \\
& >\gamma\left(f x_{m(s)-1}, f x_{n(s)-1}, \frac{\varepsilon_{1}}{c}\right) \min \{1-\lambda, 1-\lambda\} \\
& \geq 1-\lambda .
\end{aligned}
$$

This is a contradiction, therefore $\left\{x_{n}\right\}$ is a Cauchy sequence in the complete Menger probabilistic partial metric space. Hence, $u \in X$ exists such that $\lim _{m, n \rightarrow \infty} F_{x_{m}, x_{n}}(t)=\lim _{n \rightarrow \infty} F_{x_{n}, u}(t)=F_{u, u}(t)$. Now, we show that $u$ is a fixed point of $f$. We have

$$
F_{f u, u}(2 t) \geq T\left(F_{f u, x_{n}}(\phi(r)), F_{x_{n}, u}(2 t-2 \phi(r)), F_{x_{n}, x_{n}}(\phi(r)) .\right.
$$

By continuity of $\phi, r>0$ exists such that $t>\phi(r)$. Furthermore, for arbitrary $\delta \in(0,1), n_{0} \in \mathbb{N}$ exists such that for all $n \geq n_{0}$, we get

$$
F_{x_{n}, u}(2 t-2 \phi(r))>1-\delta .
$$

Hence, from (2.15), (2.19) and (2.20), we obtain that

$$
F_{f u, u}(2 t) \geq T\left(F_{f u, x_{n}}(\phi(r)), 1-\delta, 1-\delta\right)
$$

Since $\delta>0$ is arbitrary and $T$ is continuous, we can write $F_{f u, u}(2 t) \geq F_{f u, x_{n}}(\phi(r))$. Without loss of generality we may assume that $x_{n} \neq f u$ for all $n \in \mathbb{N}$, otherwise if for infinitely many values of $n, x_{n}=f u$, then $u=f u$, and hence the proof is finished. Applying (2.14) and (iv), we derive

$$
\begin{aligned}
F_{u, f u}(2 t) & \geq F_{x_{n}, f u}(\phi(r)) \\
& \geq \beta\left(x_{n-1}, u, r\right) F_{f x_{n-1}, f u}(\phi(r)) \\
& \geq \gamma\left(f x_{n-1}, f u, \frac{r}{c}\right) \min \left\{F_{x_{n-1}, f x_{n-1}}\left(\phi\left(\frac{r}{c}\right)\right), F_{u, f u}\left(\phi\left(\frac{r}{c}\right)\right)\right\} .
\end{aligned}
$$


Letting $n \rightarrow \infty$ in the above inequality, we get $F_{f u, u}(\phi(r)) \geq F_{u, f u}\left(\phi\left(\frac{r}{c}\right)\right)$. Thus $u=f u$ by Lemma 2.1. Hence $f$ has a fixed point. Moreover, if $(\mathrm{H})$ holds, then by using a similar technique as in the proof of Theorem 2.4 one can see that $u$ is a unique fixed point of $f$.

\section{Application to integral equation}

Here, in this section, we wish to study the existence of a solution to a Volterra type integral equation, as an application of our results.

Let $k>0$ be an arbitrary fixed number. Consider the following Volterra type integral equation:

$$
x(t)=g(x(t))+\int_{0}^{t} \Omega(t, s, x(s)) d s, t \in[0, k]
$$

where $g \in C(\mathbb{R})$ and $\Omega \in C([0, k] \times[0, k] \times \mathbb{R}, \mathbb{R})$. Equip $C([0, k], \mathbb{R})$ with the partial metric

$$
p(x, y)=\max _{t \in[0, k]}\{|x(t)|,|y(t)|\}, \quad x, y \in C([0, k], \mathbb{R}) .
$$

Now, we define the mapping $F: C([0, k], \mathbb{R}) \times C([0, k], \mathbb{R}) \rightarrow \mathcal{D}^{+}$by

$$
F_{x, y}(t)=H(t-p(x, y)), \quad t>0, \quad x, y \in C([0, k], \mathbb{R}) .
$$

We know that $\left(C([0, k], \mathbb{R}), F, T_{M}\right)$ is a complete Menger probabilistic partial metric space.

Theorem 3.1. Let $k>0, g \in C(\mathbb{R})$, and $\Omega \in C([0, k] \times[0, k] \times \mathbb{R}, \mathbb{R})$, satisfying the following conditions:

(i) $\|\Omega\|_{\infty}=\sup _{t, s \in[0, k], x \in C([0, k], \mathbb{R})}|\Omega(t, s, x(s))|<\infty$,

(ii) $c_{1}, c_{2} \in(0,1)$ exist such that for all $x \in C([0, k], \mathbb{R})$, all $r \in \mathbb{R}$, and all $t, s \in[0, k]$ we have

$$
|g(r)| \leq c_{1}|r|,|\Omega(t, s, x(s))| \leq \frac{c_{2}}{k}|x(s)|
$$

and $c=c_{1}+c_{2}<1$. Then, the Volterra type integral equation (3.1) has a solution $x^{*} \in C([0, k], \mathbb{R})$.

Proof. Consider the complete Menger probabilistic partial metric space $\left(C([0, k], \mathbb{R}), F, T_{M}\right)$ defined as (3.2). Now, we define the mapping $f: C([0, k], \mathbb{R}) \rightarrow C([0, k], \mathbb{R})$ by

$$
f(x)(t)=g(x(t))+\int_{0}^{t} \Omega(t, s, x(s)) d s .
$$

For each $x, y \in C([0, k], \mathbb{R})$ we have

$$
\begin{aligned}
p(f(x), f(y)) & =\max _{t \in[0, k]}\{|f(x)(t),| f(y)(t) \mid\} \\
& \leq \max _{t \in[0, k]}\left\{|g(x(t))|+\int_{0}^{t}|\Omega(t, s, x(s))| d s,|g(y(t))|+\int_{0}^{t}|\Omega(t, s, y(s))| d s\right\} \\
& \left.\leq c \max _{t \in[0, k]}\{|x(t)|,|y(t)|\}\right) \\
& =c p(x, y) \\
& \leq c \max \{p(x, y), p(y, f(y)), p(y, f(x))\} .
\end{aligned}
$$


Applying (3.2), for any $r>0$, we derive

$$
\begin{aligned}
F_{f(x), f(y)}(r) & =H(r-p(f(x), f(y))) \\
& \geq H\left(\frac{r}{c}-\max \{p(x, y), p(y, f(y)), p(y, f(x))\}\right) \\
& =\min \left\{F_{x, y}\left(\frac{r}{c}\right), F_{y, f(y)}\left(\frac{r}{c}\right), F_{y, f(x)}\left(\frac{r}{c}\right)\right\}
\end{aligned}
$$

for all $x, y \in C([0, k], \mathbb{R})$. Thus all conditions of Theorem 2.3 are satisfied, when $\phi(r)=r$ for all $r>0$ and $\beta(x, y, t)=\gamma(x, y, t)=1$ for all $x, y \in C([0, k], \mathbb{R})$ and $t>0$. Therefore, $f$ has a fixed point $x^{*} \in C([0, k], \mathbb{R})$, which is the solution of the integral equation (3.1).

Example 3.1. Consider the following Volterra type integral equation

$$
x(t)=\frac{1}{3} \cos (x(t))+\int_{0}^{t} \frac{\ln \left(t+s+\frac{1}{2}\right) \sin \left(e^{-3 t} x(s)\right)}{3 \cosh \sqrt[5]{2 s+1}} d s .
$$

Observe that Eq. (3.3) is a special case of the Eq. (3.1) when $g(r)=\frac{1}{3} \cos (r)(r \in \mathbb{R})$, and $\Omega(t, s, x(s))=$ $\frac{\ln \left(t+s+\frac{1}{2}\right) \sin \left(e^{-3 t} x(s)\right)}{3 \cosh \sqrt[5]{2 s+1}}(t, s \in[0,1])$. Obviously, all the conditions of Theorem 3.1 are satisfied. Then the Volterra type integral equation (3.3) has at least one solution which belongs to the space $C([0,1], \mathbb{R})$.

\section{NUMERICAL RESULTS}

Numerical methods can help us to investigate the solutions of differential and integral equations (see for instance $[2,11,18])$. In this section, we use a numerical method to find an approximate solution for a Volterra type integral equation. For this purpose, we use successive approximations method (SAM) [2] to find a solution for the Example 3.1.

The SAM, also called the Picard iteration method provides a scheme that can be used for solving initial value problems or integral equations. This method solves any problem by finding successive approximations to the solution by starting with an initial guess as $x_{0}(t)$, called the zeroth approximation. As will be seen, the zeroth approximation is any selective real-valued function that will be used in a recurrence relation to determine the other approximations. The most commonly used values for the zeroth approximations are 0 , 1 , or $t$. Of course, other real values can be selected as well.

Given the nonlinear Volterra type integral equation

$$
x(t)=f(x(t))+\lambda \int_{a}^{t} K(t, s, x(s)) d s,
$$

where $x$ is the unknown function to be determined, $K(t, s, x(s))$ is the kernel, and $\lambda$ is a parameter. The successive approximation method introduces the recurrence relation

$$
\begin{gathered}
\widetilde{x_{0}}(t)=\text { any selective real valued function, } \\
\widetilde{x_{n+1}}(t)=f\left(\widetilde{x_{n}}(t)\right)+\lambda \int_{a}^{t} K\left(t, s, \widetilde{x_{n}}(s)\right) d s, n \geq 0 .
\end{gathered}
$$


The solution is determined by using the limit

$$
\widetilde{x}(t)=\lim _{n \rightarrow \infty} \widetilde{x_{n+1}}(t) .
$$

Now, we discuss on the solutions of Example 3.1. To this end, consider the following nonlinear equation

$$
x(t)=F(x(t))+G(x(t)),
$$

where

$$
F(x(t))=\frac{1}{3} \cos (x(t))
$$

and

$$
G(x(t))=\int_{0}^{t} \frac{\ln \left(t+s+\frac{1}{2}\right) \sin \left(e^{-3 t} x(s)\right)}{3 \cosh \sqrt[5]{2 s+1}} d s .
$$

Applying SAM, we solve the nonlinear Volterra type integral equation (3.3). To this aim, put $t=0$ in equation (3.3). We get $x(0)=\frac{1}{3} \cos (x(0))$ or equivalently $\alpha=\frac{1}{3} \cos (\alpha)$. It gives us $\alpha=0.3176508287$. Now, we choose $\widetilde{x_{0}}(t)=0.3176508287$ in (4.1), by doing two steps of successive approximations method we find $\widetilde{x_{2}}(t)$ and consider it as an approximation for $x(t)$ (see Fig. 1). Since equation (3.3) is nonlinear, it is difficult to proceed this method further. In order to see how good is this approximation, we put $\widetilde{x_{2}}(t)$ in the left and right hand sides of equation (3.3) instead of $x(t)$, and consider the difference of these values as error. we define

$$
\operatorname{err}_{S A M}(t):=\left|\widetilde{x_{2}}(t)-\left(F\left(\widetilde{x_{2}}(t)\right)+G\left(\widetilde{x_{2}}(t)\right)\right)\right| .
$$

In table (1), we have calculated $\widetilde{x_{2}}(t)$ and $\operatorname{err}_{S A M}(t)$ at different values of $t$. The error graph of $\operatorname{err}_{S A M}(t)$ is also plotted in Figure (2) in the interval $[0,1]$.

TABLE 1. The values of $\widetilde{x_{2}}(t)$ and error related to SAM for different values of $t$

\begin{tabular}{|r|r|r|}
\hline $\mathrm{t}$ & $\widetilde{u_{2}}(t)$ & $\operatorname{err} r_{S A M}(t)$ \\
\hline 0 & $3.17 \mathrm{e}-1$ & $\mathrm{e}-10$ \\
0.1 & $3.14 \mathrm{e}-1$ & $2.93 \mathrm{e}-5$ \\
0.2 & $3.15 \mathrm{e}-1$ & $2.86 \mathrm{e}-5$ \\
0.3 & $3.16 \mathrm{e}-1$ & $1.23 \mathrm{e}-5$ \\
0.4 & $3.17 \mathrm{e}-1$ & $1.1 \mathrm{e}-5$ \\
0.5 & $3.19 \mathrm{e}-1$ & $3.66 \mathrm{e}-5$ \\
0.6 & $3.21 \mathrm{e}-1$ & $6.22 \mathrm{e}-5$ \\
0.7 & $3.23 \mathrm{e}-1$ & $8.66 \mathrm{e}-5$ \\
0.8 & $3.24 \mathrm{e}-1$ & $1.09 \mathrm{e}-4$ \\
0.9 & $3.25 \mathrm{e}-1$ & $1.31 \mathrm{e}-4$ \\
1 & $3.27 \mathrm{e}-1$ & $1.51 \mathrm{e}-4$ \\
\hline
\end{tabular}




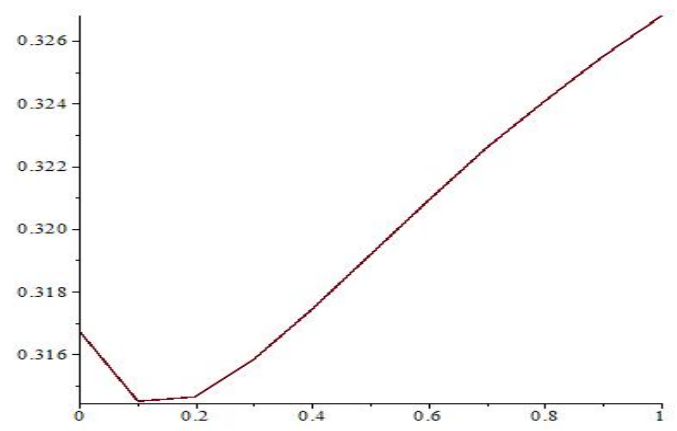

FIGURE 1. graph of approximate solution $\widetilde{x}$ in $[0,1]$

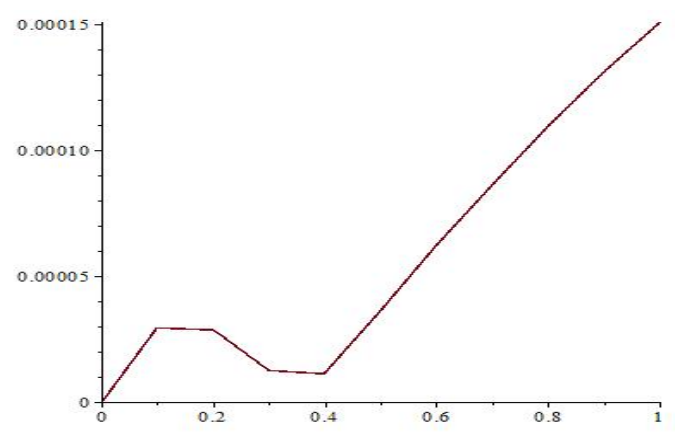

FigURE 2. absolute error graph of approximate solution $\widetilde{x}$ in $[0,1]$

\section{REFERENCES}

[1] T. Abdeljawada, E. Karapinar and K. Taş, Existence and uniqueness of a common fixed point on partial metric spaces, Appl. Math. Lett. (2011), 1-5.

[2] W. Abdul-Majid, Linear and Nonlinear Integral Equations: Methods and Applications, Springer, 2011.

[3] H. Aydi, E. Karapinar and S. Rezapour, A Generalized Meir-Keeler contraction on partial metric spaces, Abstr. Appl. Anal. 2012 (2012), Article ID 287127.

[4] N.A. Babaĉev, Nonlinear generalized contraction on Menger PM-spaces, Appl. Anal. Discrete Math. 6 (2012), $257-264$.

[5] M. Bukatin, R. Kopperman, S. Matthews and H. Pajoohesh, Partial metric spaces, Am. Math. Montly, 116 (2009), $708-718$.

[6] S. Chauhan, S. Bhatnagar and S. Radenović, Common fixed point theorems for weakly compatible mappings in fuzzy metric spaces, Le Mathematiche, LXVIII (2013)-Fasc. I, 87-98.

[7] S. Chauhan, M. Imdad, C. Vetro and W. Sintunavarat, Hybrid coincidence and common fixed point theorems in Menger probabilistic metric spaces under a strict contractive condition with an application, Appl. Math. Comput. 239 (2014), $422-433$.

[8] T. Došenović, D. Rakić, B. Carić and S. Radenović, Multivalued generalizations of fixed point results in fuzzy metric spaces, Nonlinear Anal., Model. Control, 21(2) (2016), 211-222.

[9] P.N. Dutta, B.S. Choudhury and K.P. Das, Some fixed point results in Menger spaces using a control function, Surv. Math. Appl. 4 (2009), 41-52. 
[10] D. Gopal, M. Abbas and C. Vetro, Some new fixed point theorems in Menger PM-spaces with application to Volterra type integral equation, Appl. Math. Comput. 232 (2014), 955-967.

[11] L. Grammont, Nonlinear integral equations of the second kind: A new version of Nyström method. Numer. Funct. Anal. Optim. 34(5) (2013), 496-515.

[12] O. Hadžić and E. Pap, Fixed point theory in probabilistic metric spaces, Kluwer Academic Publishers, 2001.

[13] F. Hasanvand and M. Khanehgir, Some fixed point theorems in Menger $P b M$-spaces with an application, Fixed Point Theory Appl. 2015 (2015), 81.

[14] S.G. Matthews, Partial metric topology, Proc. 8th Summer Conference on General Topology and Applications. Ann. N.Y. Acad. Sci. 728 (1994), 183-197.

[15] K. Menger, Statistical metrics, Proc. Nat. Acad. Sci. USA, 28 (1942), 535-537.

[16] Z. Mustafa, J. Rezaei Roshan, V. Parvaneh and Z. Kadelburg, Some common fixed point results in orderd partial $b$-metric spaces, J. Inequal. Appl. 2013 (2013), 562.

[17] P. Patle, D. Patel, H, Aydi and S. Radenović, On $\mathcal{H}^{+}$type multivalued contraction and its applications in symmetric and probabilistic spaces, Mathematics, 7 (2019), 144.

[18] M. Rabbani and R. Arab, Extension of some theorems to find solution of nonlinear integral equation and homotopy perturbation method to solve it, Math. Sci. 11(2) (2017), 87-94.

[19] S. Romaguera, A Kirk type characterization of completeness for partial metric spaces, Fixed Point Theory Appl. 2010 (2010), Article ID 493298, 6 pages.

[20] B. Schweizer and S. Sklar, Statistical metric spaces, Pacific J. Math. 10 (1960), 313-334.

[21] B. Schweizer and A. Sklar, Probabilistic Metric Spaces, Elsevier, North-Holland, New York, 1983.

[22] S. Sedghi, N. Shobkolaei, T. Došenović and S. Radenović, Suzuki-type of common fixed point theorems in fuzzy metric spaces, Math. Slovaca 68(2) (2018), 451-462.

[23] V.M. Sehgal and A.T. Bharucha-Reid, Fixed point of contraction mappings in PM-spaces, Math. Syst. Theory, 6 (1972), $97-102$.

[24] Y. Shi, L. Ren and X. Wang, The extension of fixed point theorems for set valued mapping, J. Appl. Math. Comput. 13 (2003), 277-286.

[25] Stevens, Metrically generated PM-spaces, Fund. Math. (1968), 259-269.

[26] Y. Su and J. Zhang, Fixed point and best proximity point theorems for contractions in new class of probabilistic metric spaces, Fixed Point Theory Appl. 2014 (2014), 170. 